\title{
Methods
}

\section{Fluorescence Resonance Energy Transfer (FRET) imaging}

To monitor the PKA activity in the developing rat retina, we transfected P2 rat retinas with the genetically encoded fluorescence-based reporter, A-kinase activity reporter (AKAR3), consisting of the fusion of cyan fluorescent protein (CFP), a phosphothreonine-binding domain (FHA1), a PKA consensus phosphorylation target, and the yellow fluorescent protein (YFP) variant citrine [44,45,46,47,48,49,50,51,52]. In response to phosphorylation by PKA, this PKA activity reporter undergoes conformational changes to bring the CFP and the YFP variant in a close position; thus, the ratios of fluorescence resonance energy transfer (FRET) were increased [13,44,45,46,47,48,49,53,54] (Figure S3-Cii-Ciii).

Live FRET imaging was performed on an upright fluorescent microscope (Olympus BX51WI) using a 60× water immersion objective (Olympus LUMPLFLW). During FRET imaging, the retinal explants expressing AKAR3 were continuously superfused with oxygenated ACSF at $30^{\circ} \mathrm{C}$. To acquire the FRET signal, CFP was excited by a narrow bandwidth (436/10 nm). Two emission wavelengths were collected simultaneously using a Dual-View image splitter (Optical Insights) with appropriate YFP (535/30 nm) and CFP (480/40 nm) emission filters. Images were captured with a CCD camera (CoolSNAP HQ2, Photometrics) at 2 s-intervals, with exposure times ranging from 250 to 500 ms. Digitized imaging data were subsequently analyzed by MetaMorph, with background subtraction from both channels and the correction of CFP bleed-through into the YFP channel according to the previous study [13].

FRET ratios were calculated as $\mathrm{R}=\left(\mathrm{F}_{\mathrm{YFP}} / \mathrm{F}_{\mathrm{CFP}}\right)$ for individual cells (Figure S3-CiiCiii). Before, during and after drug application, the values of the FRET ratio were calculated by averaging over five images around the maximum response (Figure S3-DiDii). The magnitude of drug-induced change was reported as the change in FRET ratios $(\Delta \mathrm{R})$, computed by subtracting the value of the FRET ratio before drug application from the value of the FRET ratio around the maximum response to drug (Figure S3-E). 


\section{Pharmacology}

The selective $A_{2 A} R$ agonist (CGS 21680) or antagonist (ZM 241385) and the PKA inhibitor (H89) were purchased from Tocris Biosciences and prepared as 1000× concentrated stock solutions in Dimethyl sulfoxide (DMSO). Stocks were stored at $-20^{\circ} \mathrm{C}$ and diluted in ACSF on the day of the experiments. Drug application was delivered by a perfusion system. 